\title{
Current Concepts in the Mandibular Condyle Fracture Management Part I: Overview of Condylar Fracture
}

\author{
Kang-Young Choi, Jung-Dug Yang, Ho-Yun Chung, Byung-Chae Cho \\ Department of Plastic and Reconstructive Surgery, Kyungpook National University School of Medicine, Daegu, Korea
}

\begin{abstract}
The incidence of condylar fractures is high,but the management of fractures of the mandibular condyle continues to be controversial. Historically, maxillomandibular fixation, external fixation, and surgical splints with internal fixation systems were the techniques commonly used in the treatment of the fractured mandible. Condylar fractures can be extracapsular or intracapsular, undisplaced, deviated, displaced, or dislocated. Treatment depends on the age of the patient, the co-existence of other mandibular or maxillary fractures, whether the condylar fracture is unilateral or bilateral, the level and displacement of the fracture, the state of dentition and dental occlusion, and the surgeonnds on the age of the patient, the co-existence of othefrom which it is difficult to recover aesthetically and functionally;an appropriate treatment is required to reconstruct the shape and achieve the function ofthe uninjured status. To do this, accurate diagnosis, appropriate reduction and rigid fixation, and complication prevention are required. In particular, as mandibular condyle fracture may cause long-term complications such as malocclusion, particularly open bite, reduced posterior facial height, and facial asymmetry in addition to chronic pain and mobility limitation, great caution should be taken. Accordingly, the authors review a general overview of condyle fracture.
\end{abstract}

Keywords Mandibular condyle / Mandibular fractures / Temporomandibular joint
Correspondence: Kang-Young Choi Department of Plastic and Reconstructive Surgery, Kyungpook National University School of

Medicine, 130 Dongduk-ro, Jung-gu, Daegu 700-721, Korea

Tel: +82-53-420-5685

Fax: +82-53-425-3879

E-mail:kychoi@knu.ac.kr

This article was invited as part of a panel presentation, which was one of the most highly rated sessions by participants, at the 69th Congress of the Korean Society of Plastic and Reconstructive Surgeons on November 11, 2011 in Seoul, Korea.

No potential conflict of interest relevant to this article was reported.

Received: 24 Jun 2012 • Revised: 25 Jun 2012 • Accepted: 26 Jun 2012

pISSN: 2234-6163 • elSSN: 2234-6171 • http://dx.doi.org/10.5999/aps.2012.39.4.291 • Arch Plast Surg 2012;39:291-300

\section{INTRODUCTION}

Among facial bone fractures, the mandible fracture has a highest incidence next to nasal bone fracture and condyle fracture most frequently occurs in mandible fracture [1]. Condyle fracture accounts for approximately $30 \%$ and $37 \%$ of mandible fracture in dentulous mandible patients and edentulous mandible patients, respectively. The reason for a high incidence of mandibular condyle fracture is attributable to the binding of the mandibular ramus with high stiffness and mandibular condyle head with low stiffness [2]. This is generally caused by indirect force that is delivered to the mandibular condyle head. The most common external causative factor is physical trauma, and car accident, violence, industrial hazard, fall, sports, and gunshot wound are also included in the external causative factors. Internal causative factors include osteomyelitis, benign or malignant tumor, and muscular spasm during electric shock treatment.

As mandibular fracture may cause disorders that is hard to be recover aesthetic and functionally, an appropriate treatment is required to reconstruct the shape and function of uninjured status. To do this, accurate diagnosis, appropriate reduction and rigid fixation, and complication prevention are required. In

Copyright $(\odot 2012$ The Korean Society of Plastic and Reconstructive Surgeons

This is an Open Access article distributed under the terms of the Creative Commons Attribution Non-Commercial License (http://creativecommons.org/

licenses/by-nc/3.0/) which permits unrestricted non-commercial use, distribution, and reproduction in any medium, provided the original work is properly cited.

www.e-aps.org 
particular, as mandibular condyle fracture may cause long-term complications such as mandibular growth and functional disorders, and chronic temporomandibular joint (TMJ) complication, a more caution should be given. Accordingly, the authors review the general overview on condyle fracture here.

\section{ANATOMY AND PHYSIOLOGY OF MANIDIBLE}

\section{The mandible}

The mandible, which is the hardest monostotic bone among facial bones, is a U-shaped long bone (Fig. 1). It consists of areas with and without teeth, and binds to the bilateral temporal bones at the left and right TMJ. At the early stage of development, it develops laterally and fuses at the midline 1 to 2 years after birth, forming a complete structure like the maxilla. Name of each area has some variation depending on the literatures. However, the most clinically useful classification of each area divides the mandible into the symphysis and parasymphysis, body, angle and ramus, condylar process, coronoid process, and alveolar process. The symphysis and parasymphysis refers to an area between both canine. The body refers to an area from the canine to the second molar. The angle and ramus refers to the area next to third molar except for the coronoid and condylar process. The mandibular condyle consists of the condylar process and head of the mandible. The condylar process and head subunit refers to a superior area of the extension line connecting the masseteric tuberosity from the deepest area of the sigmoid notch. The condylar process and head subunit consists of the head, neck, and subcondylar area. These are three height level lines that divide the subunit, and define the boundary. The three height level lines consist of an extension line that is parallel to the posterior border of the mandible, an extension line that parallelly heads for the perpendicular from the deepest area of the sigmoid notch, and an extension line that is inferior to the condylar head lateral pole. The condylar head refers to an area that is superior to the extension line that is inferior to the condylar head lateral pole. The condylar neck refers to an area between the extension line that is inferior to the condylar head lateral pole and the extension line that parallelly heads for the perpendicular from the deepest area of the sigmoid notch. The subcondylar area refers to an area that is inferior to the extension line that parallelly heads for the perpendicular from the deepest area of the sigmoid notch. Meanwhile, the condylar neck is divided into the high level and low level, and the reference line dividing them is an extension line that is positioned in the middle of the sigmoid notch line and the lateral pole line of the head (Fig. 1).

The elevated area of the midline of the mandible is defined as mental protuberance. The mental tubercle exists inferior to the left and right elevated areas and the mental foramen by which important facial nerves and blood vessels pass exists laterally superior to the aforementioned nodule area. Internally, a pair of the mental spines exists inferiorly to the midline, from which the genio-glossus and stylohyoid muscle originate. The mylohyoid line that reaches the mandibular ramus postero-superiorly exists at the lateral side of the mental spine. The mylohyoid muscle originates from the mylohyoid line. The nerve root is positioned below the mylohyoid muscle, and controls it. The mandibular angle and ramus is a rectangular bony part that exists superoposteriorly to the mandibular body. Mandibular angle size varies depending on age and individuals. It is approximately $140^{\circ}$ in children and $110^{\circ}$ to $120^{\circ}$ in adults. The tuberositas masseterica and tuberositas pterygoidea exist at the lateral side and internal side of mandibular angle, respectively. They are insertion parts of the masseter muscle and medial pterygoid muscle, respectively.

\section{Temporo-mandibular joint}

The mandibular condyle forms articulation with the mandibular fossa of the temporal bone, which is called temporo mandibular joint. The articulation disc, which is a fibrous tissue, exists

\section{Fig. 1. The anatomy of mandibular area}

(A) Anterior view of mandible. (B) The subregions of the condylar process. The mandible is composed body, angle, ramus, symphysis and parasymphysis, condylar process, coronoid process. The condylar process and head is a subunit of the mandible and is defined by an oblique line running backward from the sigmoid notch to the upper masseteric tuberosity.
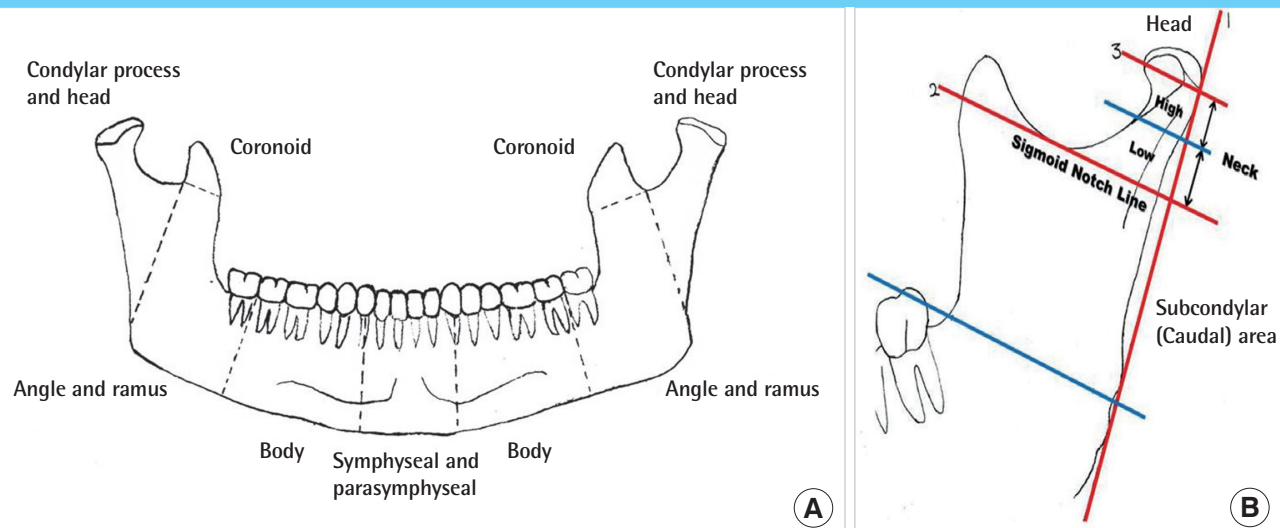
between the TMJ, and acts as a buffer. As synovial fluid that has lubrication function inside the joint capsule exists around the joint. It minimizes friction during joint motion, and enables the smooth movement of the joint. The articulation disc generally has an oval shape. However, as its shape is determined by the shape of the condyle head and mandibular fossa, its size and shape may vary. The articulation disc is composed of fibrous tissues without nerve or blood vessel, and disc thickness is $1 \mathrm{~mm}$ for the center, $3 \mathrm{~mm}$ for the posterior aspect, and $2 \mathrm{~mm}$ for the anterior aspect. Thus, it has a shape that is the thinnest in the center and the thickest in the posterior aspect. For the normal joint, the mandibular condyle is positioned at the center which is the thinnest. Thus, disc injury occurs at the center due to aging or chronic physical stimuli. The disc is attached to the condyle head via the bilateral articular disc ligaments, which prevents the deviation of the disc from the condyle head during mandibular movement. The joint capsule that surrounds the TMJ produces the synovial fluid internally, and the produced synovial fluid acts as a lubricant during mandibular movement, and supplies nutrients to an area without blood vessels. In addition, as the joint capsule has substantial wrinkles, it changes the contact area during mandibular movement, protecting the joint. The temporo mandibular ligament with a triangular shape exists at the lateral side of the articular capsule, and it prevents excessive movement that is beyond the normal range of the mandible during madibular movement. The temporo mandibular ligament is rigidly fixed by the temporal bone and mandible, which provides the stability of TMJ movement. Unlike hinge-typed joints of the four extremities, the TMJ has characteristics of the simultaneous movement of the bilateral joints during mandibular movement such as talking or eating. The left and right TMJ can not move independently, and is closely associated with dental occlusion
(Fig. 2).

\section{Occlusion and mandibular movement Occlusion}

Occlusion is intercuspation between the teeth. It is divided into static occlusion without jaw movement and functional or dynamic occlusion with jaw movement. Static occlusion may vary depending on condyle location, among which maximum intercuspal occclusion or intercuspal occlusion position refers to a site that contact the teeth most frequently. This is known as centric occlusion ( $\mathrm{CO})$. $\mathrm{CO}$ is a relationship between the teeth regardless of condyle position. As $\mathrm{CO}$ may be changed by prosthodontic treatment or jaw surgery, condyle location that is always constant and reproducible is required. This is because the condyle location that acts as a reference for prosthodontic treatment or jaw surgery is required. Procedures where the stability of condyle position is not maintained eventually cause TMJ disease or malocclusion. Based on the aforementioned concept, occlusion at condyle position that is stable and reproducible without intercupation is defined as centric relation (CR), and called centric relation occlusion (CRO). If CRO is same as $\mathrm{CO}$, it is considered as ideal occlusion. Condyle position in CR has been controversial over a long period of time. Currently, most anterior-superior position reported by Celenza and Nasedkin [3] is considered the closest position (Fig. 3).

This static occlusion occurs for a short time in a daily living. In most cases, functional occlusion with jaw movement occurs. During jaw movement anterior movement, occlusal contact occurs at the anterior teeth and disclusion occurs at the remaining teeth. During lateral movement, occlusion occurs at the working sided canine or canine premolar group teeth, and disclusion occurs at the remaining teeth. This is called guidance (Fig. 4).

\section{Fig. 2. The anatomy of temporo-mandibular joint}

(A) TMJ state at mouth closing (B) TMJ state at mouth opening. The TMJ is a hinged synovial joint, and is an articulation of the head of the mandible with the mandibular fossa and articular tubercle of the temporal bone. There are 2 movements which occur in the TMJ. First, the head of the mandible rotates anteriorly, secondly, the head of the mandible glides anteriorly as it continues to rotate anteriorly. TMJ, temporomandibular joint .

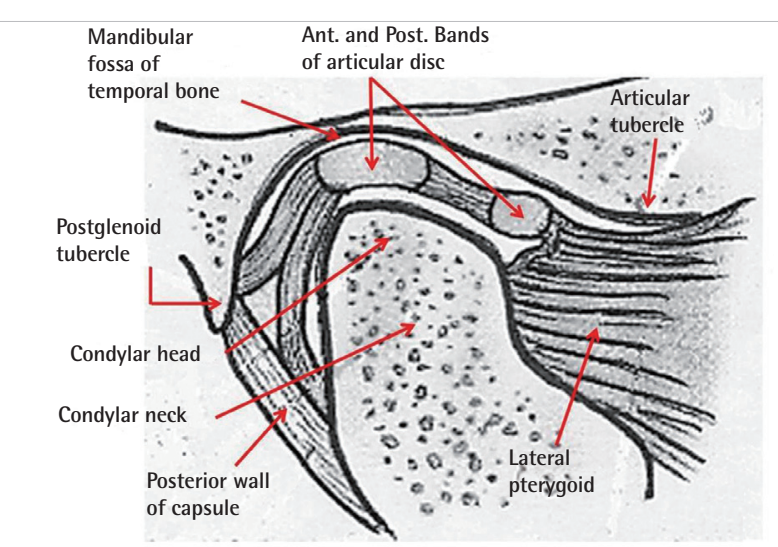

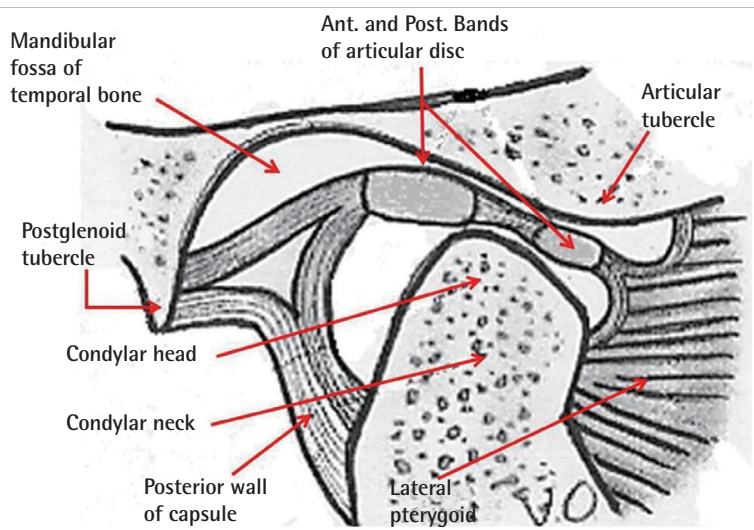

(B) 
The anterior region is far away from force point so that it is tolerable to lateral force during anterior movement. Meanwhile, the canine has a long root and the alveolar bone is hard so that it is tolerable to lateral force during lateral movement. If teeth other than the guidance teeth contact during the aforementioned

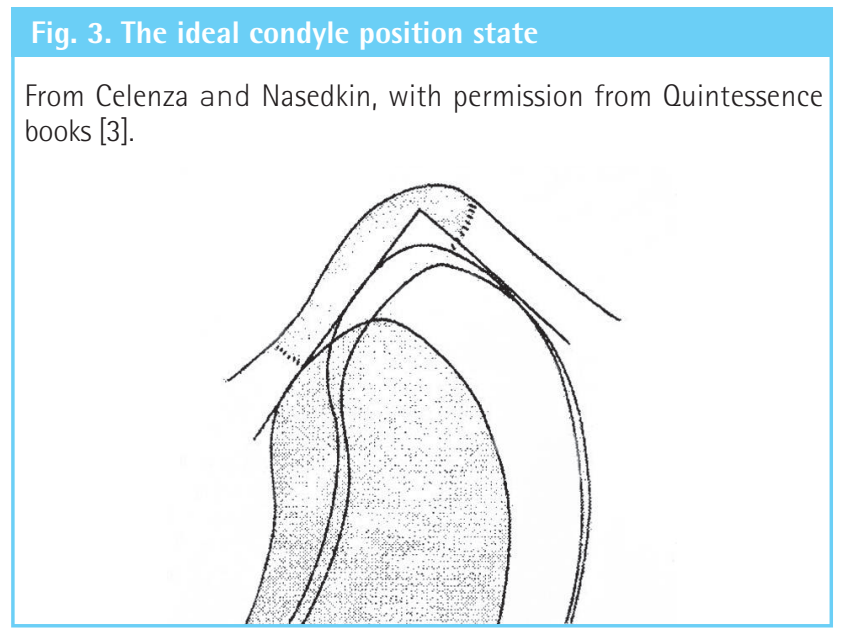

movement, they are relatively more vulnerable to lateral force. It causes periodontal ligament injury, which is vulnerable to periodontitis. Eventually, teeth supporting becomes weak due to absorption by the adjacent alveolar bone. The teeth become exfoliated. This status refers to trauma from occlusion (TFO).

\section{Mandibular movement}

Mandibular movement occurs by the involvement of the TMJ as a posterior guidance, and teeth as an anterior guidance. The TMJ as a ginglymoarthroidal joint has a movement of 25 to $30 \mathrm{~mm}$ by the upper joint space. The mouth opening as a hinge joint has rotation movement. Beyond that, the mouth opening as sliding joint has a movement in addition to rotation movement (Fig. 5). In addition, the bodily lateral movement of the mandible occurs during lateral excursions, which refers to bennett movement (Fig. 6). If no bennett movement occur due to interference caused by changes in condyle position during fracture or jaw surgery though occlusion is normal, mandibular movement disorder occurs. If the disorder is persistent for a long time, TMJ disease

\section{Fig. 4. The sequence of functional occlusion}

(A) Centric occlusion. (B) Anterior guidance. (C) Canine guidance.
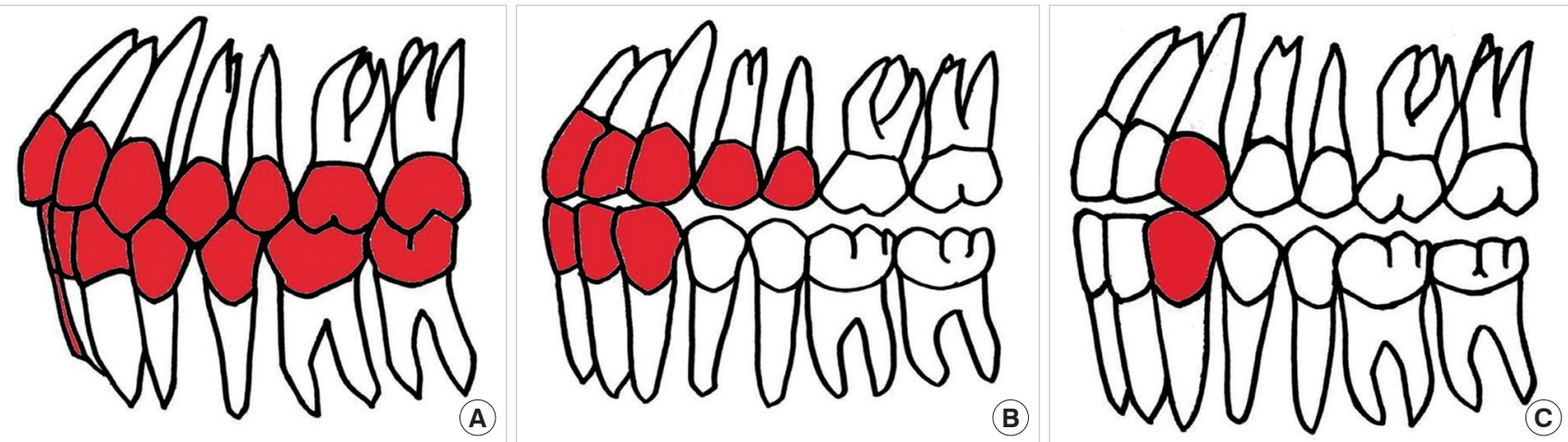

Fig. 5. Movement of the mandibular condyle

(A) Rotation movement of the condyle. (B) Translation movement of the condyle.
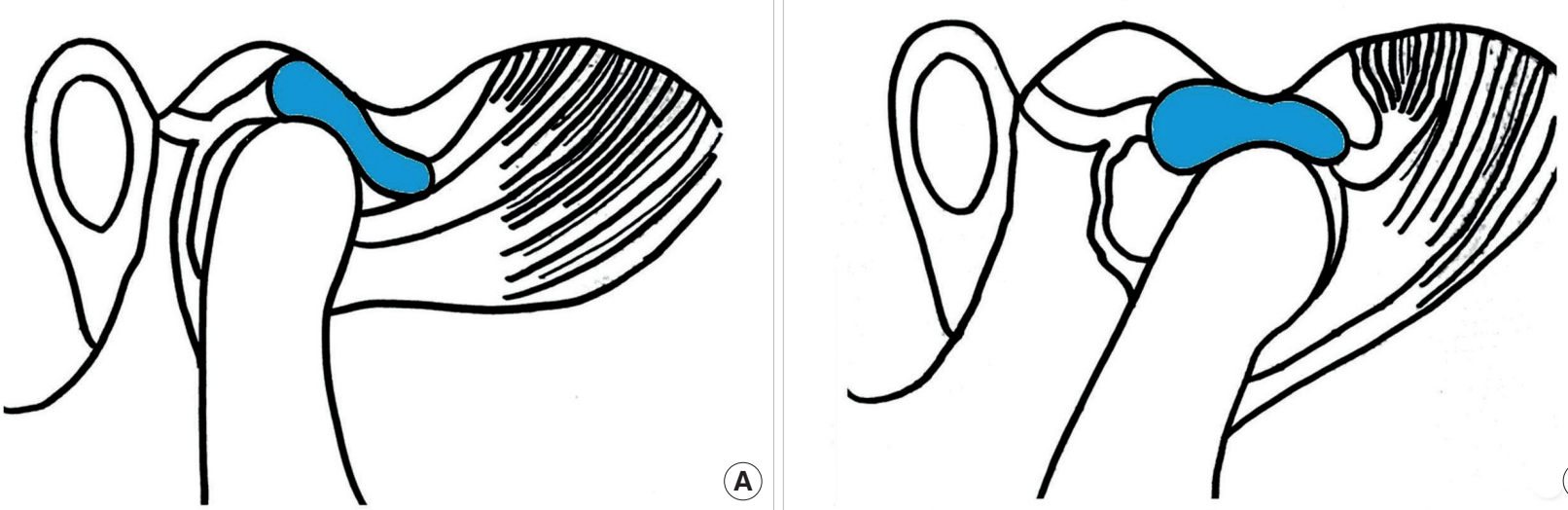
occlusion instability relapse may be caused.

\section{CLASSIFICATION OF MANDIBULAR CONDYLE FRACTURE}

Condyle fracture can be classified as follows according to Lindahl classification. It is classified into condyle head fracture, condyle neck fracture, and subcondyle fracture according to facture position. Condyle head fracture is also called intracapsular fracture as the joint capsule exists until the condyle neck. condyle neck fracture, which occurs at the inferior attach area of the joint capsule, refers to an area that becomes narrow from the condyle head. It is an extracapsular fracture as it is not included in the joint capsule, and exists at the inferior attach area of the lateral pterygoid. Subcondyle fracture, which exists inferiorly to the condyle neck, refers to an area between the mandibular sigmoid notch and mandibular posterior aspect. According to the degree of fracture fragment displacement, condyle fracture is classified into non-displaced fracture that has fracture with no displacement of the fracture site, deviated fracture where fracture fragments are displaced but some of them contact the mandibular joint, displaced fracture where fracture fragment is separated from the mandibular body and displaced, but exists in the TMJ, and dislocation where the condyle head is deviated from the TMJ (Fig. 7). The displacement of fracture fragment is observed in condyle fracture. It put mainly in traction by the masseter muscle, lateral pterygoid muscle, and temporalis muscle. The most commonly observed type is the displacement of the condyle head to the anteromedial side, which is shown in a fracture that occurs inferiorly to the lateral pterygoid muscle (Fig. 7).

\section{TREATMENT OF MANDIBULAR CONDYLE FRACTURE}

\section{Closed reduction and functional therapy}

For closed reduction, intermaxillary fixation is conducted using arch bar and wire, followed by maintaining of the fixation of the maxilla and mandible for 2 to 4 weeks. After achieving stable union of the factored site, a wire for intermaxillary fixation is removed. Then, normal occlusion is induced after fixation us-

\section{Fig. 6. Movement of the mandibular condyle}

(A) Axial view of the mandibular movement. Antero-lateral condylar movement. (B) Axial view of the mandibular movement. Lateral condylar movement. (C) Coronal view of the mandibular movement.

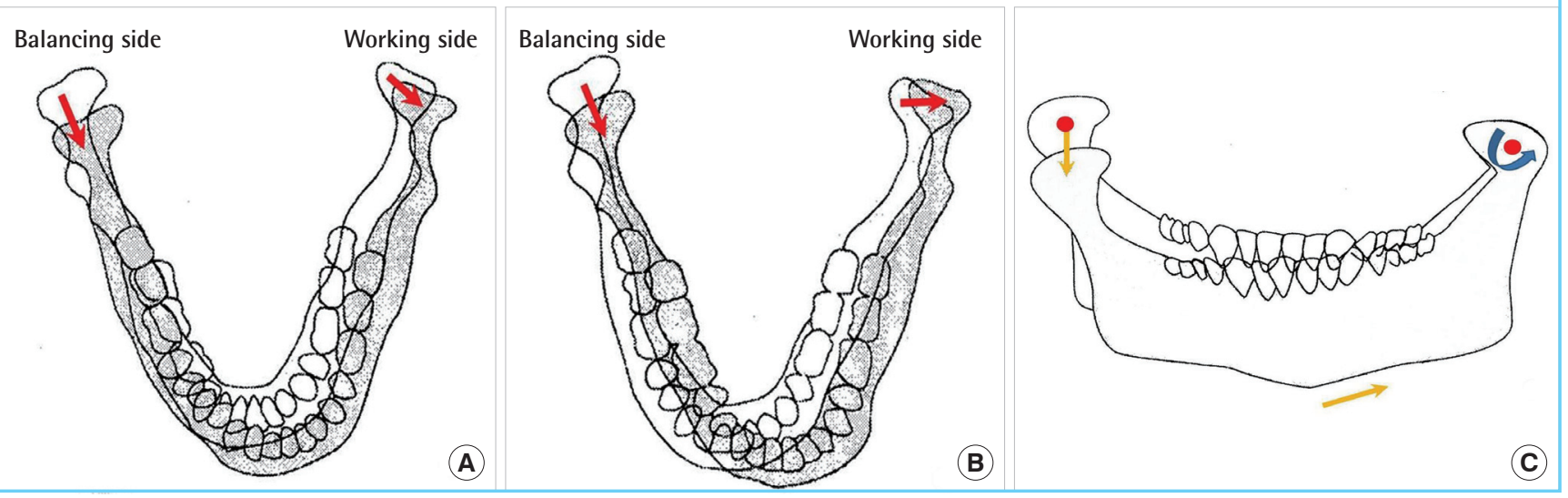

Fig. 7. The classification of mandibular condyle fracture according to Lindahl classification

(A) The degree of fracture fragment. (B) Two type of the displacement of condyle proximal segment.

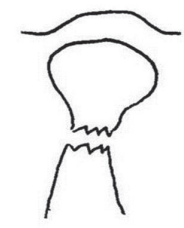

A. No displacement

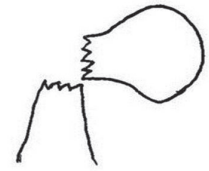

D. Deviation-Dislocation

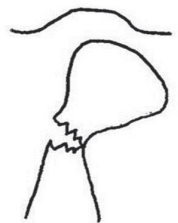

B. Deviation
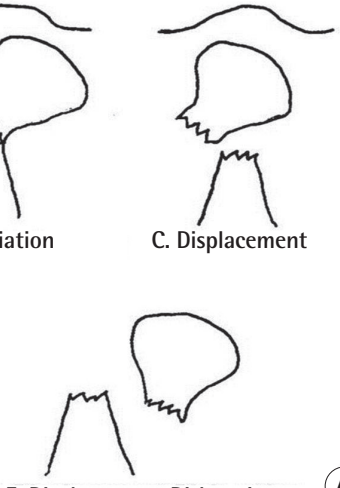

E. Displacement-Dislocation

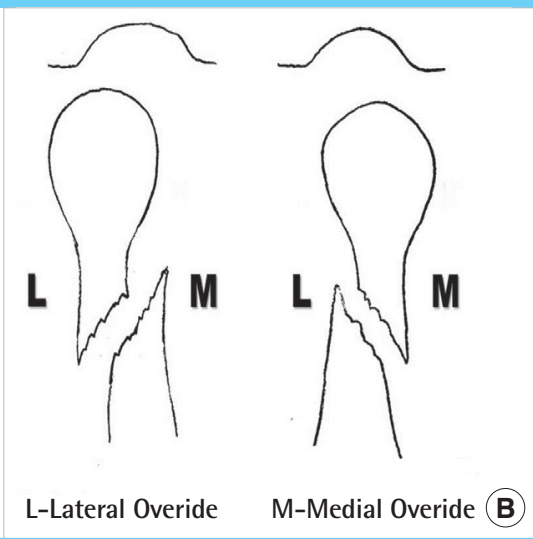


ing rubber, and soft diet is maintained for 2 weeks. Functional therapy that consists of passive mandibular movement exercise and mouth opening exercise is conducted and then clinical outcomes are observed.

\section{Open reduction and internal fixation}

There are various operation methods of open reduction for madibular condyle fracture depending on fracture site and degree of bone fragment displacement. In general, they include preauricular approach, postauricular approach, submandibular approch, Risdon approach, combined approach, and retromandibular approach. Treatment type should be selected considering patient's age, preference, fracture type, fracture of other sites, and teeth status.

\section{COMPLICATION OF MANDIBULAR CONDYLE FRACTURE}

\section{Non-union and malunion}

Non-union is associated with fracture fragment stability, repeated trauma, infection, inappropriate reduction, multiple fractures, mandibular atrophy, and patient compliance [4]. Infection of the fracture site more facilitates fibroblast growth than the activities of osteoblasts or osteoclasts. This makes fibrous tissues dominant in the bone healing area, and causes fibrous binding, which results in non-union [5]. In the case of excessive exposure of the periosteum, delayed fracture treatment is shown, particularly, in comminuted fracture and edentulous fracture. If fractured fragments are inappropriately assigned, and teeth alignment is inappropriately positioned. In a severe case, non-union may occur. Malocclusion is caused by facial asymmetry. If this happens, correction should be performed via orthognathic surgery and bone transplantation.

\section{Malocclusion}

Malocclusion is associated with patient's teeth status, fracture type, displacement of the bone segments, incomplete reduction of bone fragments, inappropriate fixation and fixation time, delayed treatment, and patient compliance [6-8]. For malocclusion that occurs after the completion of bone union, occulsal adjustment is required or even re-operation and orthodontic surgery is required in a severe case. Open reduction should be conducted in an early phase after injury to prevent malocclusion after mandibular condylar trauma. Furthermore, accurate reduction and fixation during the surgery and regular follow-up after the surgery are required to prevent postoperative malocclusion (Fig. 8).

\section{Temporo-mandibular joint dysfunction}

Temporo-mandibular joint derangement

If the functional disorders of the condyle-disc complex occur with changes in the shape of the disc due to trauma, this is called temporo-mandibular joint derangement. The functional disorders of the condyle-disc complex occurs cause disc displacement. This displacement is the most important characteristics of temporo-mandibular joint derangement, which occurs with major symptoms such as joint sound, abnormal movement of the condyle head, impingement of condyle head, mandibular movement limitation and joint pain. For temporo-mandibular joint

\section{Fig. 8. A 21-year-old woman with iatrogenic condyle fracture during facial bone contouring surgery}

(A) Preoperative 3D head computed tomography (CT). Anterior open bite was observed. The non-union or malunion will be possible. (B) Postoperative 3D head CT. The iatrogenic subcondylar fracture was corrected by open reduction and internal fixation immediately. Anterior open bite was corrected.
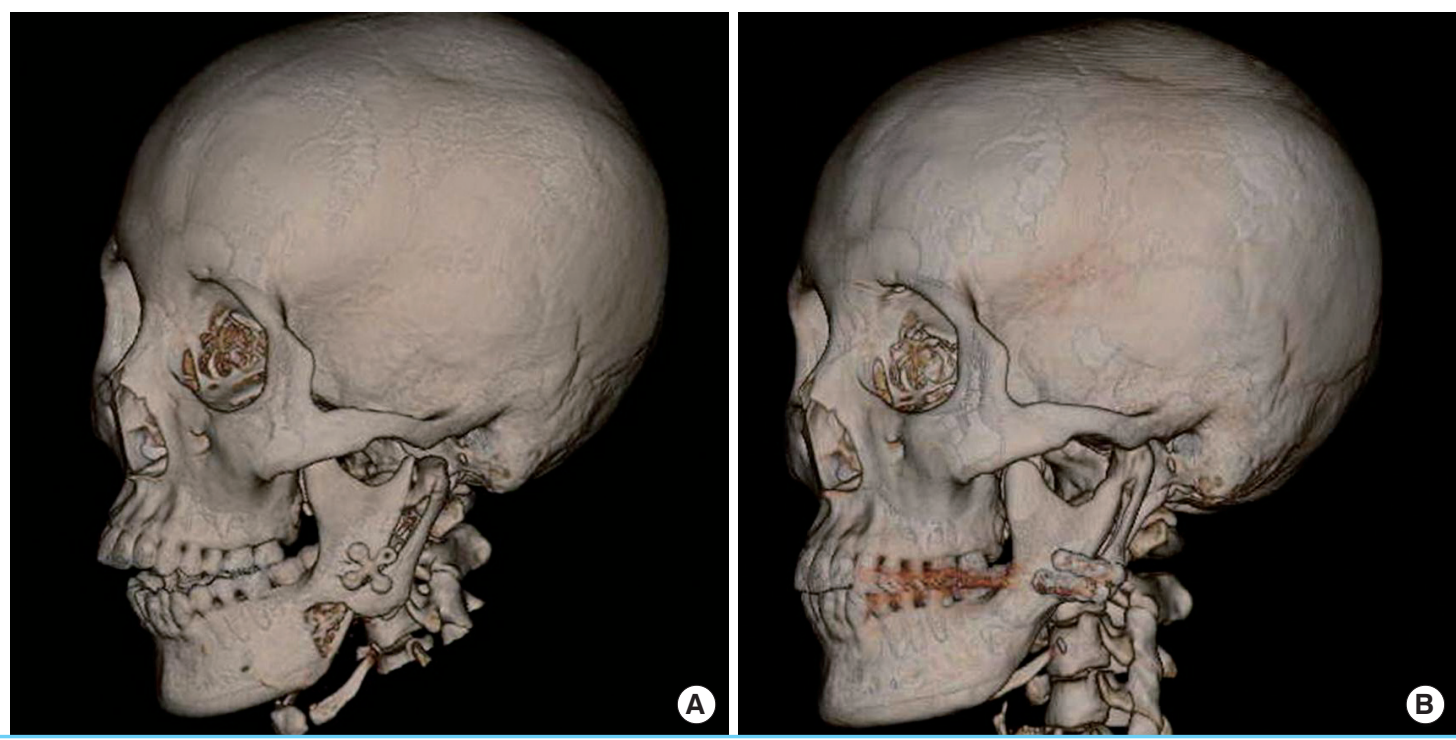
derangement, changes in disc shape and the functional disorders of the condyle-disc complex are minimized and prevented by the early reduction and rigid fixation of the displaced bone fragments adjacent to the joint (Fig. 9).

\section{Traumatic arthritis of temporo-mandibular joint}

Traumatic arthritis refers to an arthritis that occurs secondarily after the deformity of the joint due to the direct injury to the articular cartilage or fracture caused by trauma. Traumatic arthritis generally occurs after fracture invading the articular surface, but also occurs by repeated mild trauma or joint injury caused by chronic load. In particular, for intracapsular fracture among mandibular condyle fractures, articular surface injury occurs at the time of trauma onset, and traumatic arthritis is further expected to occur due to chronic and repeated joint movement. Clinical symptoms that may occur in an early phase include joint sound and pain during joint movement. As bony arthritis progresses, progressed clinical findings such as joint sound increase, lock sensation, pain, and mouth opening limitation are observed [9].

\section{Ankylosis of temporo-mandibular joint}

Ankylosis of temporo-mandibular joint refers to a movement disorder of the TMJ caused by the growth of fibrous tissues or bone tissues in the TMJ structure due to various factors. It causes severe functional and structural abnormalities in patients. Ankylosis of temporo-mandibular joint is mainly divided into two types. One is true ankylosis of temporo-mandibular joint where joint ankylosis is attributable to the joint itself. The other is pseudo ankylosis of temporo-mandibular joint where causative factors are attributable to others except for the joint. In addition, ankylosis of temporo-mandibular joint is classified into complete and partial types, and unilateral and bilateral types according to the intensity and position of ankylosis of temporo-mandibular joint, respectively. Ankylosis of temporo-mandibular joint frequently occurs by trauma. It occurs in all age brackets, but mainly occurs in patients aged less than 10 years. It has clinical symptoms such as poor nutritional status due to mouth opening or masticatory disorders, severe injury of teeth and periodontal tissues due to inappropriate oral hygiene, facial asymmetry of the adjacent TMJ, dislocation of mandible at the injured site, the tooth malalignement of the injured site, neuromuscular occlusion, shortness of mandibluar ramus, deep antegonial notch, loss and decrease of the joint capsule, and increased fracture density of the joint area [10]. If bilateral ankylosis of temporo-mandibular joint occurs at growth stage, aesthetic disorder such as micrognathia and facial asymmetry may occur due to decreased mandibular growth (Fig. 10).

\section{Growth disorder and facial asymmetry}

It has been known that decreased mandibular growth occurs in $20 \%$ to $25 \%$ of patients with mandibular condyle fracture. This growth disorder has been reported to be attributable to direct condyle growth disorder and severe functional disorder caused by the adjacent muscular stiffness, injury of soft tissues, and scar. In particular, as mandibular condyle, which is a secondary growth point that is affected by external factors such as perimandibular growth and biodynamic force, has special anatomical structures and many functions, mandibular condyle fracture

\section{Fig. 9. A 24-year-old woman with iatrogenic condyle fracture during orthognatic surgery}

Open reduction and internal fixation was done. (A) Axial view of 3D head computed tomography (CT). (B) Panorama plain film. 3D head CT and panorama was checked at postoperative 7 weeks. The distal fracture segment was displaced medially and bone gap was checked $1.84 \mathrm{~mm}$. In this case, the non-union or malunion will be occurred.
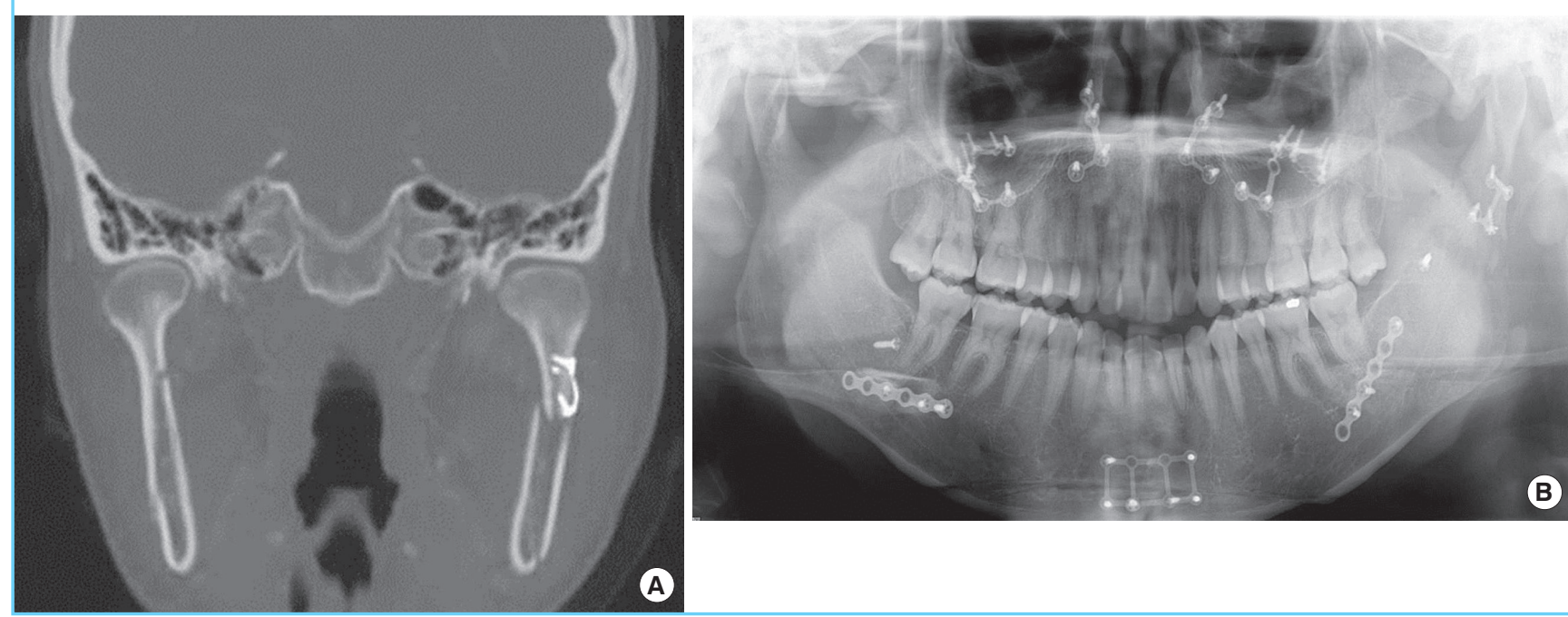
Fig. 10. An 18-year-old man with mandibular ankylosis at right side due to previous facial trauma

Inappropriate bony reduction was done at immediate trauma and the ankylosis of mandible was developed. (A) Preoperative gross photo. (B) Preoperative 3D head computed tomography (CT). (C) Preoperative panorama plain film. (D) The intraoperative mouth opening was checked $45 \mathrm{~mm}$. (E) The intraoperative grossphoto was taken. (F) Immediate postoperative gross photo. (G) Postoperative gross photo at 5 months. (H) Postoperative 3D head CT. (I) Postoperative Panorama plain film.
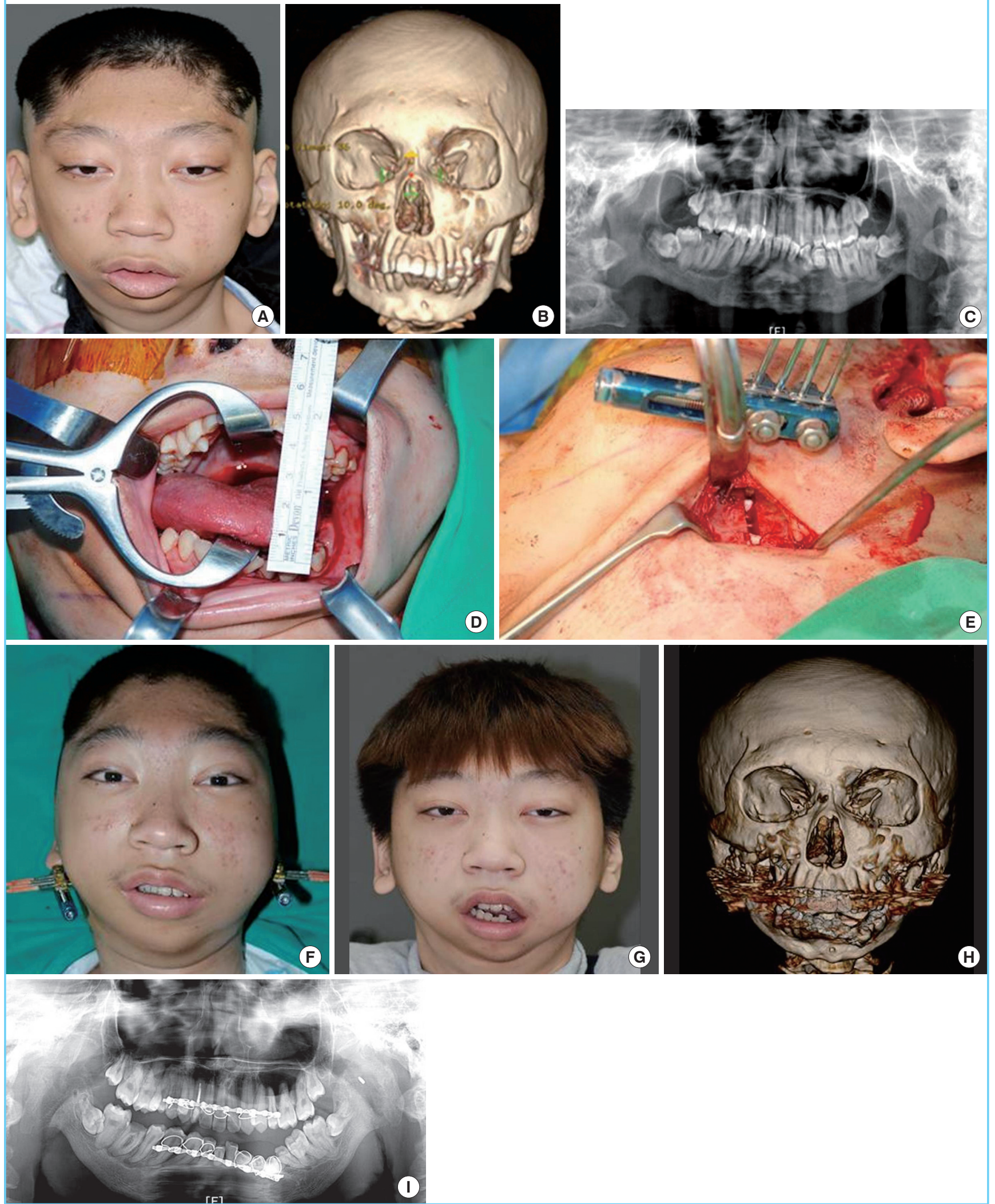
may cause the growth disorder of fracture site. In addition, condyle growth is progressed over the longest period among other parts of the craniofacial, at which mandibular asymmetry occurs due to lateral growth, traumatic and neuronal disorders. As the mandible supports the soft tissues of the lower face, changes in its position or shape are important for facial asymmetry.

\section{Condyle resorption}

Condylar resorption, a TMJ's non-functional remodeling, is also known as condylar dissolution. It is defined as a status where condyle shape change and size decrease gradually occur [11]. Despite the successful reduction of condyle fracture, excessive dissection and injury of the adjacent blood vessels during the surgery may cause condyle head resorption over a long period. As a result, patients may complain of the instability of occlusion and skeleton, TMJ dysfunction, and pain, and facial asymmetry, anterior open bite, and mandibular setback may occur [12]. For example, Nam's method, where osteotomy is conduced on condyle fracture at subcondyle level to pull out the fragment, and then put it back to its original position after reduction, was previously used as a surgery method for condyle fracture. However, due to resorption caused by poor condylar vascularization, it is no longer used.

\section{Nerve injury}

Various open reduction approaches have been used to treat madibular fracture. Nerve injury may occur during flap elevation, fracture reduction, and insertion of a metal plate $[13,14]$. Axonotmesis, which is a main nerve injury, causes sensory loss for 4 weeks or more. It takes approximately 4 weeks to recover from neuropraxia. In particular, a precaution should be given not to cause the injury of facial nerve temporal branch during preauricular approach. If nerve injury is observed after the surgery, steroid should be immediately administered to prevent tissue swelling, thereby shortening recovery time and preventing permanent injury.

\section{Infection}

Infection should be prevented by the accurate reduction of the displaced bone fragments at the early stage of mandibular fracture. After the surgery, pulp vitality test of the fracture line or adjacent teeth, and the periodontal status should be carefully followed-up. In addition, an early treatment is required to minimize complications caused by infection. Many studies reported that infection occurred in $7 \%$ of patients with mandibular fracture [15]. In addition, some studies reported preoperative infection, most of which are associated with delayed early treatment and the teeth positioned on the fracture line. Postoperative infection may occur by various factors. In particular, bone segment instability, patient's systemic status, fracture degree; foreign materials, open window, and preoperative and postoperative oral hygiene with a risk of contamination in the fracture site are important causative factors. Thus, infection should be prevented by the accurate reduction of the displaced bone fragments at the early stage of mandibular fracture. After the surgery, pulp vitality test of the fracture line or adjacent teeth, and the periodontal status should be carefully followed-up to minimize complication caused by infection.

\section{CONCLUSIONS}

The treatment of mandibular condyle fractures depends on the biologic character and adaptive capability of the masticatory system. These will differ widely among patients, and it is the lack of sound biology and adaptation that can lead to an unfavorable outcome. Therefore we must understand the functional mechanism of the mandible. Furthermore, the successful treatment of mandibular fractures may be accomplished by a variety of techniques when one adheres to sound surgical principles related to the diagnosis, stable fixation, and rehabilitation of the patient. The recovery of patient's pre-injury occlusion and function is the ultimate goal when treating fractures of the mandible. To obtain this ultimate goal, accurate diagnosis, appropriate reduction and rigid fixation, and complication prevention are required.

\section{REFERENCES}

1. Turvey TA. Midfacial fractures: a retrospective analysis of 593 cases. J Oral Surg 1977;35:887-91.

2. Fridrich KL, Pena-Velasco G, Olson RA. Changing trends with mandibular fractures: a review of 1,067 cases. J Oral Maxillofac Surg 1992;50:586-9.

3. Celenza FV, Nasedkin JN. Occlusion: the state of the art. Chicago: Quintessence Pub. Co.; 1978.

4. Mathog RH, Toma V, Clayman L, et al. Nonunion of the mandible: an analysis of contributing factors. J Oral Maxillofac Surg 2000;58:746-52.

5. Mathog RH. Nonunion of the mandible. Otolaryngol Clin North Am 1983;16:533-47.

6. Moreno JC, Fernandez A, Ortiz JA, et al. Complication rates associated with different treatments for mandibular fractures. J Oral Maxillofac Surg 2000;58:273-80.

7. Champy M, Lodde JP, Schmitt R, et al. Mandibular osteosynthesis by miniature screwed plates via a buccal approach. J Maxillofac Surg 1978;6:14-21.

8. Passeri LA, Ellis E 3rd, Sinn DP. Complications of nonrigid 
fixation of mandibular angle fractures. J Oral Maxillofac Surg 1993;51:382-4.

9. Hansson T, Nilner M. A study of the occurrence of symptoms of the temporomandibular joint masticatory musculature and related structure. J Oral Rehabil 1975;2:313-24.

10. Mathog RH. Maxillofacial trauma. Baltimore: Williams \& Wilkins; 1984.

11. Huang YL, Pogrel MA, Kaban LB. Diagnosis and management of condylar resorption. J Oral Maxillofac Surg 1997; 55:114-9.

12. Balasubramaniam R, Van Sickels J, Falace D. Condylar re- sorption following temporomandibular joint arthroscopy in a patient with essential thrombocythemia. Oral Surg Oral Med Oral Pathol Oral Radiol Endod 2006;101:581-7.

13. Cawood JI. Small plate osteosynthesis of mandibular fractures. Br J Oral Maxillofac Surg 1985;23:77-91.

14. Dodson TB, Perrott DH, Kaban LB, et al. Fixation of mandibular fractures: a comparative analysis of rigid internal fixation and standard fixation techniques. J Oral Maxillofac Surg 1990;48:362-6.

15. James RB, Fredrickson C, Kent JN. Prospective study of mandibular fractures. J Oral Surg 1981;39:275-81. 